\title{
Interplay between nonstandard and nuclear constraints in coherent elastic neutrino-nucleus scattering experiments
}

\author{
B. C. Cañas,,$^{1, *}$ E. A. Garcés $\odot^{2, \dagger}$ O. G. Miranda, ${ }^{3, \ddagger}$ A. Parada, ${ }^{4, \S}$ and G. Sanchez Garcia $\odot^{3, \|}$ \\ ${ }^{1}$ Universidad de Pamplona, Km 1, vía salida a Bucaramanga, Campus Universitario, \\ 543050 Pamplona, Colombia \\ ${ }^{2}$ Instituto de Física, Universidad Nacional Autónoma de México, Apdo. \\ Postal 20-364, CDMX 01000, México \\ ${ }^{3}$ Departamento de Física, Centro de Investigación y de Estudios Avanzados del IPN, Apdo. \\ Postal 14-740, 07000 Ciudad de México, México \\ ${ }^{4}$ Facultad de Ciencias Básicas, Universidad Santiago de Cali, Campus Pampalinda, \\ Calle 5 No. 6200, 760035 Santiago de Cali, Colombia
}

(Received 3 December 2019; accepted 16 January 2020; published 10 February 2020)

\begin{abstract}
New measurements of the coherent elastic neutrino-nucleus scattering (CEvNS) are expected to be achieved in the near future by using two neutrino production channels with different energy distributions: the very low energy electron antineutrinos from reactor sources and the muon and electron neutrinos from spallation neutron sources (SNS) with a relatively higher energy. Although precise measurements of this reaction would allow for an improved knowledge of standard and beyond the Standard Model physics, it is important to distinguish the different new contributions to the process. We illustrate this idea by constraining the average neutron root mean square (rms) radius of the scattering material, as a standard physics parameter, together with the nonstandard interactions (NSI) contribution as the new physics formalism. We show that the combination of experiments with different neutrino energy ranges could give place to more robust constraints on these parameters as long as the systematic errors are under control.
\end{abstract}

DOI: 10.1103/PhysRevD.101.035012

\section{INTRODUCTION}

Four decades after its theoretical prediction [1], the COHERENT Collaboration [2] has eventually accomplished the challenge of first observing the coherent elastic neutrino-nucleus scattering (CEvNS) phenomenon. This measurement was achieved with neutrinos coming from a spallation neutron source (SNS) with energies up to $52.8 \mathrm{MeV}$. The importance of this neutrino reaction lays on the fact that it can be used as a powerful tool for precision measurements at low energy ranges, as well as a mechanism for ruling out or confirming a variety of new physics scenarios [3-26].

In addition, future dark matter experiments are nearing their sensitivity to the neutrino floor [27], and an accurate

\footnotetext{
*blanca.canas@unipamplona.edu.co †egarces@ fisica.unam.mx ¥omr@fis.cinvestav.mx

§alexander.parada00@usc.edu.co

"gsanchez@fis.cinvestav.mx
}

Published by the American Physical Society under the terms of the Creative Commons Attribution 4.0 International license. Further distribution of this work must maintain attribution to the author(s) and the published article's title, journal citation, and DOI. Funded by SCOAP ${ }^{3}$. characterization of the detector target materials through CEvNS [28], using diverse neutrino data from SNS and reactors, will be important in discriminating the neutrino background from a true wimp signature $[29,30]$.

The CEvNS reaction in the energy regime of the SNS depends on nuclear form factors, introducing their own source of errors $[29,30]$, which unfortunately tend to be difficult to have under control. In contrast, the detection of CEvNS from reactor neutrinos (with an average energy of $\left\langle E_{\nu}\right\rangle \approx 4 \mathrm{MeV}$ ) in the foreseeable future, would allow the study of neutrino physics in the very low energy window, a regime where there is nearly no contribution of the nuclear form factor. Many experiments under commission are in the quest for the first detection of CEvNS using reactor neutrinos as a source; among them we have TEXONO [31], CONUS [32], NU-CLEUS [33], CONNIE [34-38], MINER [39], RED100 [40], and RICOCHET [41].

Because of their different characteristics, it can be expected that the combination of CEvNS from a reactor and SNS fluxes can accurately constrain standard and nonstandard physics. It would be desirable to investigate how the correlation between different parameters describing standard and nonstandard interactions can be disentangled combining various experimental setups. The interplay among different observables has been analyzed in previous studies, including the correlation between the weak mixing 
angle and the neutron skin [42], as well as between the form factor uncertainties and new physics constraints in current and future SNS CEvNS experiments [43]. On the other hand, in this work, we simultaneously study the potential to measure the relevant parameter for the nuclear form factor (the neutron rms radius $R_{n}$ ) and the restriction to new physics in the nonstandard interactions (NSI) formalism, a model independent picture able to describe many beyond Standard Model scenarios for neutrino interactions [44-47]. Until now, NSI have been extensively studied in the context of CEvNS [12,13,15,25,26,48-50], and its current constraints are already useful for global analysis [51-54], specially to break some of the well-known degeneracy problems leading to the LMA-dark solution $[55,56]$ and to the possible degeneracy in probing $C P$ violation in neutrino oscillations $[57,58]$.

\section{NONSTANDARD NEUTRINO INTERACTIONS AND CEVNS}

As we mentioned above, the detection of CEvNS has been pursued for a long time, and since the first results were presented by the COHERENT Collaboration [2], the interest to confirm such reaction has increased. In this section, we briefly introduce the main characteristics of the CEvNS process, to be used in later calculations. Within the SM, the CEvNS differential cross section is given by [59-62]

$$
\left(\frac{d \sigma}{d T}\right)_{\mathrm{SM}}^{\mathrm{coh}}=\frac{G_{F}^{2} M}{\pi}\left[1-\frac{M T}{2 E_{\nu}^{2}}\right]\left[Z g_{V}^{p} F_{Z}\left(q^{2}\right)+N g_{V}^{n} F_{N}\left(q^{2}\right)\right]^{2},
$$

where, $M$ is the mass of the nucleus, $E_{\nu}$ the incoming neutrino energy, $T$ is the nucleus recoil energy, $F_{Z, N}\left(q^{2}\right)$ are the nuclear form factors, and the neutral current vector couplings (including radiative corrections) are given by [48]

$$
\begin{aligned}
& g_{V}^{p}=\rho_{\nu N}^{N C}\left(\frac{1}{2}-2 \hat{\kappa}_{\nu N} \hat{s}_{Z}^{2}\right)+2 \lambda^{u L}+2 \lambda^{u R}+\lambda^{d L}+\lambda^{d R} \\
& g_{V}^{n}=-\frac{1}{2} \rho_{\nu N}^{N C}+\lambda^{u L}+\lambda^{u R}+2 \lambda^{d L}+2 \lambda^{d R},
\end{aligned}
$$

where $\rho_{\nu N}^{N C}=1.0086, \hat{s}_{Z}^{2}=\sin ^{2} \theta_{W}=0.2312, \hat{\kappa}_{\nu N}=0.9978$, $\lambda^{u L}=-0.0031, \lambda^{d L}=-0.0025$, and $\lambda^{d R}=2 \lambda^{u R}=7.5 \times 10^{-5}$ [63]. Notice that the previous relations depend on fundamental parameters such as the weak mixing angle, $\sin \theta_{W}$, on nuclear physics through the form factors $F_{Z}\left(q^{2}\right)$ and $F_{N}\left(q^{2}\right)$, and on the specific detection target through the proportion of protons to neutrons $Z / N$.

For the purposes of this work, we are also interested in the search for new physics. As mentioned before, a common framework is that of nonstandard neutrino interactions [44-46]. In this scenario, new terms containing nonuniversal and flavor changing currents are present. These terms are parametrized as dimensionless coefficients $\varepsilon_{\alpha \beta}^{q V}$ (with $q=u, d, V=L, R$, and $\alpha, \beta=e, \mu, \tau$.) proportional to the Fermi constant. The parameters for which $\alpha=$ $\beta$ refer to nonuniversal interactions, while those with $\alpha \neq \beta$ correspond to flavor-changing terms. By introducing these parameters, the CEvNS cross section in the spinless limit, for $T \ll E_{\nu}$, is given by $[32,48,50,64-66]$

$$
\begin{aligned}
\frac{\mathrm{d} \sigma}{\mathrm{d} T}\left(E_{\nu}, T\right) \simeq & \frac{G_{F}^{2} M}{\pi}\left(1-\frac{M T}{2 E_{\nu}^{2}}\right) \\
& \times\left\{\left[Z\left(g_{V}^{p}+2 \varepsilon_{e e}^{u V}+\varepsilon_{e e}^{d V}\right) F_{Z}^{V}\left(Q^{2}\right)\right.\right. \\
& \left.+N\left(g_{V}^{n}+\varepsilon_{e e}^{u V}+2 \varepsilon_{e e}^{d V}\right) F_{N}^{V}\left(Q^{2}\right)\right]^{2} \\
& +\sum_{\alpha}\left[Z\left(2 \varepsilon_{\alpha e}^{u V}+\varepsilon_{\alpha e}^{d V}\right) F_{Z}^{V}\left(Q^{2}\right)\right. \\
& \left.\left.+N\left(\varepsilon_{\alpha e}^{u V}+2 \varepsilon_{\alpha e}^{d V}\right) F_{N}^{V}\left(Q^{2}\right)\right]^{2}\right\},
\end{aligned}
$$

in the expression above, we have exemplified NSI for the case of an incoming electron (anti)neutrino flux, as is the case of reactor neutrino experiments. The corresponding cross section for an incident muon and tau (anti)neutrino is straightforward to obtain by replacing $e \leftrightarrow \mu, \tau$, respectively. Comparing this last equation with the Standard Model case of Eq. (1), we can notice that, besides the dependence on the previous parameters, such as the nuclear form factors, we now have the dependence on the NSI parameters $\varepsilon_{\alpha \beta}^{q V}$. A first approach has been to study each of the observables separately. With this method, it has been possible to obtain constraints for the neutron rms radius $[16,43,67]$, NSI parameters $[15,68]$, and neutrino electromagnetic properties $[15,69,70]$, among others. Most of these analyses have been done for one parameter at a time. As a next step in this direction, we aim to forecast whether a combination of different CEvNS measurements disentangles information from different observables, in order to have more reliable constraints.

In the next sections, we will discuss this case of NSI as well as the neutron radius distribution, the latter being a parameter contained within the nuclear form factor.

\section{CEVNS EXPERIMENTS WITH REACTOR ANTINEUTRINOS}

Reactors have been a useful source of antineutrinos since the first neutrino signal ever detected [71,72]. The most used reaction to detect them has been the inverse beta decay [73-76] while neutrino-electron scattering has given complementary information, although with less statistics due to the smaller cross section [77,78]. A measurement of CEvNS from reactor antineutrinos will provide yet another channel to measure the antineutrino flux and could give complementary information to constrain, for instance, a sterile neutrino signal $[17,21,22]$. 
TABLE I. List of the experimental proposals to detect CEvNS in reactor neutrinos. We show the information for the expected electron recoil energy threshold $\left(T_{\text {thres }}\right)$, the distance from the source to the detector (Baseline) the detector technology, (Det. Tec), and the fiducial mass (Fid. Mass).

\begin{tabular}{lccccc}
\hline \hline & $T_{\text {thres }}$ & Baseline & $Z / N$ & Det. Tec. & Fid. Mass \\
\hline CONNIE [34,35] & $28 \mathrm{eV}$ & $30 \mathrm{~m}$ & 1.0 & CCD (Si) & $0.1-1 \mathrm{~kg}$ \\
CONUS [32] & $100 \mathrm{eV}$ & $10 \mathrm{~m}$ & 0.79 & HPGe & $4-100 \mathrm{~kg}$ \\
\hline \hline
\end{tabular}

The expected number of events to be detected in a CEvNS reactor antineutrino experiment is computed by using the integral of the incoming neutrino flux times the cross section,

$$
\begin{aligned}
N_{\text {events }}= & t \phi_{0} \frac{M_{\text {detector }}}{M} \int_{E_{\nu \text { min }}}^{E_{\nu \max }} \lambda\left(E_{\nu}\right) d E_{\nu} \\
& \times \int_{T_{\min }}^{T_{\max }\left(E_{\nu}\right)}\left(\frac{d \sigma}{d T}\right)^{\mathrm{coh}} d T,
\end{aligned}
$$

where $t$ is the exposure time of the experiment (that we will consider as one year), $\phi_{0}$ is the antineutrino flux from the reactor, $M_{\text {detector }}$ is the detector mass, $M$ is the nucleon mass, and $\lambda\left(E_{\nu}\right)$ is the antineutrino energy spectrum. Notice that, in general, the cross section $d \sigma / d T$ refers to either the Standard Model or to the NSI one, which are given, respectively, by Eqs. (1) and (3).

Due to the low energy antineutrino spectrum, the momentum transfer is small and the nuclear form factors are expected to be close to unity and almost constant. Therefore, the dependence on nuclear physics effects will be rather weak for this kind of experiment.

For the antineutrino energy spectrum $\lambda\left(E_{\nu}\right)$, we will use the theoretical one discussed in Refs. $[79,80]$, which is parametrized as

$$
\lambda\left(E_{\nu}\right)=\Sigma_{l} f_{l} \lambda_{l}\left(E_{\nu}\right)=\Sigma_{l} f_{l} \exp \left[\Sigma_{k=1}^{6} \alpha_{k l} E_{\nu}^{k-1}\right]
$$

$f_{l}$ represents the fission fraction for the given isotope $\left({ }^{235} \mathrm{U}\right.$, ${ }^{239} \mathrm{Pu},{ }^{241} \mathrm{Pu},{ }^{238} \mathrm{U}$ ) in the reactor. For the case of CONNIE, we will consider [34,35] $(0.55: 0.32: 0.06: 0.07)$, while for CONUS, we use the fission fractions [32] (0.58:0.30: $0.05: 0.07)$. For the specific values of the coefficients $\alpha_{k l}$, we follow the prescription given in [79]. This expression is valid for energies above $2 \mathrm{MeV}$, while for lower energies, we use the values reported in [81].

With this information, we can make a forecast of the sensitivity to NSI for a future CEvNS reactor antineutrino measurement. We can consider that the experiment will measure the Standard Model (SM) predicted number of events, $N^{\exp }=N_{\text {events }}^{\mathrm{SM}}$, with a given statistical and systematic error. We can then compute a $\chi^{2}$ statistical analysis as

$$
\chi^{2}=\frac{\left(N_{\mathrm{events}}^{\mathrm{SM}}-N^{t h}\right)^{2}}{\sigma_{\mathrm{stat}}^{2}+\sigma_{\mathrm{syst}}^{2}} .
$$

There are several experiments that are trying to observe CEvNS from a reactor antineutrino flux. In this work, we will focus on two particular experimental setups, the CONUS and the CONNIE proposals, for which the main characteristics are listed in Table I. One of the main reasons for considering these experiments is that they use two different materials (germanium and silicon, respectively) and detection technologies, this will lead to complementary results for the NSI constraints, specially because they have a different proportion of protons to neutrons, a quantity to which the cross section is sensitive.

After performing a $\chi^{2}$ analysis, we have found the corresponding constraints on NSI for these two proposals. In each analysis, we take only two nonzero NSI parameters.

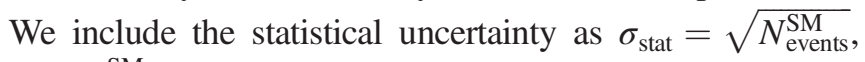
with $N_{\text {events }}^{\mathrm{SM}}$ the expected number of events to be measured in one year. As for the systematics, it is difficult to forecast what would be the final experimental error. However, for the case of CONUS [32], it has been considered that a 5\% normalization error and a 3\% other overall systematic errors can be a realistic scenario. To be more conservative, in this work, we will assume an $8 \%$ contribution for the systematic errors, considering that systematic uncertainties in quantities such as the quenching factor $(\mathrm{QF})$ can also play a significant role. For completeness, we also computed the optimistic case of a $4 \%$ systematic error and, finally, the ideal case for which only statistical uncertainties are taken into account; although it is unexpected that the systematics could be smaller than the statistical error, it gives an idea of the more optimistic achievable constraint. The results are shown in Fig. 1 for the nonuniversal NSI parameters and in Fig. 2 for the flavor-changing ones. We can see that both proposals could give a good restriction for the NSI parameters if they are considered one at a time. For the flavorchanging analysis, we have only considered the $e \tau$ channel, since the $e \mu$ NSI parameter has already been constrained with other neutrino processes [44-46]. In particular, we can see that, when we take one parameter at a time, the constraint is quite strong and would improve current limits given by other experiments [44-46]. This information is of relevance for the future exact determination of the standard oscillation parameters by long-baseline experiments, in particular of the $C P$ violating phase, $\delta_{C P}$, that could have a different value if flavor changing NSI are present [58].

Moreover, if we combine the two results, it might be possible to obtain a more robust constraint in the sense that the parameter degeneracy can be resolved. In this case, it is important to include the systematic errors of the reactor antineutrino flux and their correlations. We will see that the 

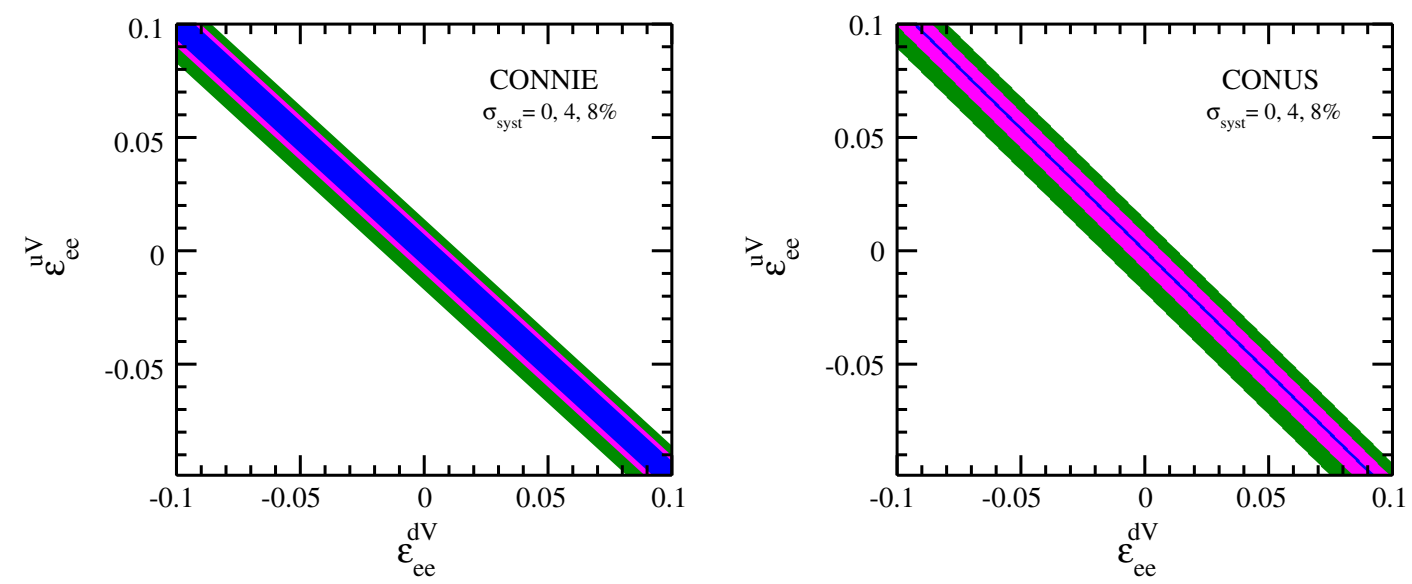

FIG. 1. Expected exclusion for the nonuniversal NSI parameters in the case of detection of CEvNS at the reactor neutrino experiments CONNIE and CONUS, the colored regions indicate the exclusion with an overall systematic error of $0 \%, 4 \%$, and $8 \%$.
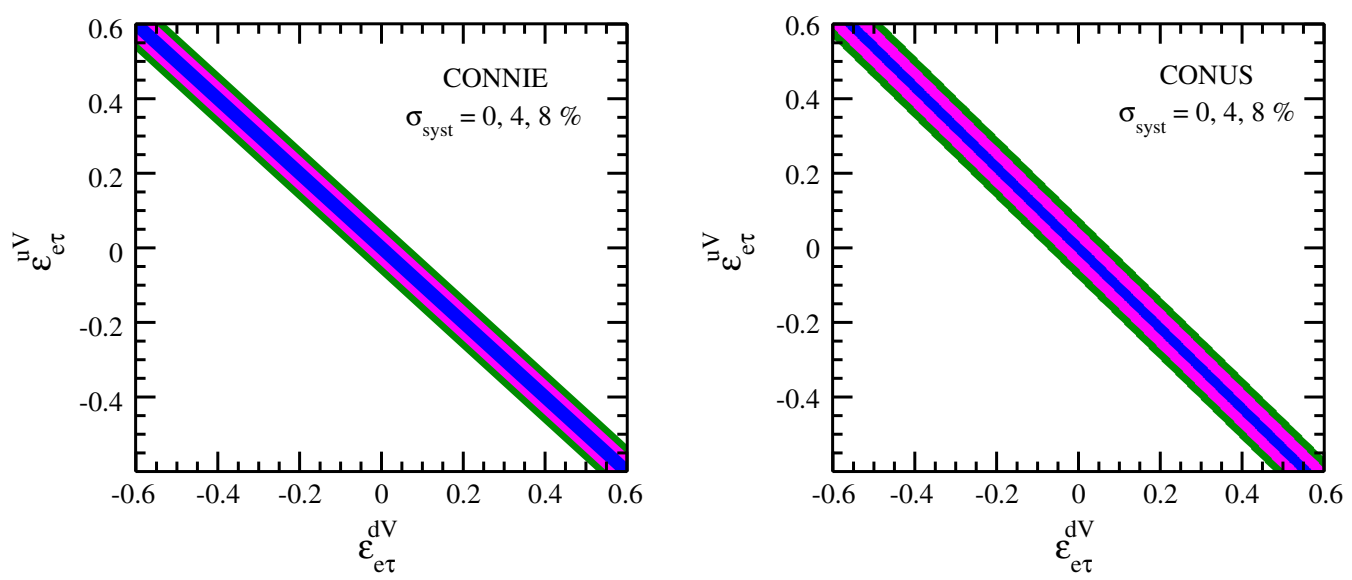

FIG. 2. Expected exclusion for the flavor changing NSI parameters in the case of detection of CEvNS at the reactor neutrino experiments CONNIE and CONUS, the colored regions indicate the exclusion with an overall systematic error of $0 \%, 4 \%$, and $8 \%$.

influence of such correlated error is mild, and perhaps there could be other systematic uncertainties that would be more relevant, such as the QF.

To illustrate the effect of the correlations for the reactor antineutrino spectrum, we have considered it for the moment as the only source of systematic uncertainty and made a complete $\chi^{2}$ analysis. For this analysis, we closely follow the approach described in Ref. [82] where the covariance matrix is diagonalized in order to simplify the numerical analysis. In this framework, we start with the covariance matrix,

$$
V_{k k^{\prime}}^{l}=\delta \alpha_{k l} \delta \alpha_{k^{\prime}} \rho_{k k^{\prime}}^{l},
$$

where $\delta \alpha_{k l}$ are the errors of the coefficients $\alpha_{k k^{\prime}}$ that appear in Eq. (5) and $\rho_{k k^{\prime}}^{l}$ the corresponding correlation matrix (these values were reported in Ref. [79]). The systematic error arising from the reactor antineutrino flux is given then by

$$
\left(\delta N_{l}^{\nu}\right)^{2}=\sum_{k k^{\prime}} \frac{\partial N_{l}^{\nu}}{\partial \alpha_{k l}} \frac{\partial N_{l}^{\nu}}{\partial \alpha_{k^{\prime} l}} V_{k k^{\prime}}^{l}
$$

To work with the diagonal form of the covariance matrix, it is necessary to introduce new coefficients [82], $c_{k l}$, that are defined by the relation,

$$
\alpha_{k l}=\sum_{k^{\prime}} \mathcal{O}_{k^{\prime} k}^{l} c_{k^{\prime} l},
$$

with $\mathcal{O}^{l}$ a rotation matrix such that it diagonalizes the covariance matrix $V^{l}$,

$$
\mathcal{O}^{l} V^{l}\left(\mathcal{O}^{l}\right)^{T}=\operatorname{diag}\left[\left(\delta c_{k l}\right)^{2}\right] .
$$

With this new parametrization, the reactor antineutrino flux will be given by

$$
\lambda_{l}\left(E_{\nu}\right)=\exp \left[\sum_{k=1}^{6} c_{k l} p_{k}^{l}\left(E_{\nu}\right)\right],
$$




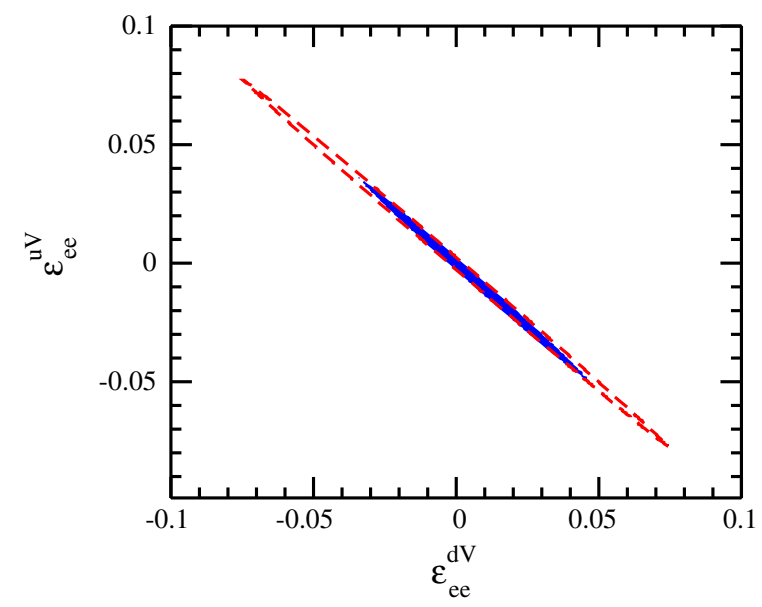

FIG. 3. Expected exclusion for the nonuniversal NSI parameters for a combined analysis of the reactor neutrino experiments CONNIE and CONUS. We include only the statistical error and the correlated systematic error from the reactor antineutrino spectrum (solid blue area). The region indicates the exclusion at $90 \%$ C.L. when we consider a $100 \%$ efficiency. For comparison, we also show the result of considering a $2 \%$ systematic error without any correlations (dashed line); the results in both cases are qualitatively similar.

with $p_{k}^{l}\left(E_{\nu}\right)$ the polynomial,

$$
p_{k}^{l}\left(E_{\nu}\right)=\sum_{k^{\prime}=1}^{6} \mathcal{O}_{k k^{\prime}}^{l} E_{\nu}^{k^{\prime}-1} .
$$

After these computations, the covariance matrix will be given as

$$
\sigma_{i j}^{2}=\Delta_{i}^{2} \delta_{i j}+\sum_{l} \delta N_{i}^{l} \delta N_{j}^{l}
$$

with $\Delta_{i}^{2}$ the statistical uncertainty for the $i^{\text {th }}$ bin either for the CONNIE or CONUS future measurement, and $\delta N_{i}^{l}$ the contribution to the systematic error of the $i^{\text {th }}$ bin due to the $l$ isotope $\left({ }^{235} \mathrm{U},{ }^{239} \mathrm{Pu},{ }^{241} \mathrm{Pu},{ }^{238} \mathrm{U}\right)$.

With all these computations, we can now define the covariance analysis for the $\chi^{2}$ function,

$$
\chi^{2}=\sum_{i j}\left(N_{i}^{\text {theo }}-N_{i}^{\text {exp }}\right) \sigma_{i j}^{-2}\left(N_{j}^{\text {theo }}-N_{j}^{\text {exp }}\right),
$$

with

$$
N_{i}^{\text {theo }}=N_{i}^{235}+N_{i}^{238}+N_{i}^{241}+N_{i}^{239},
$$

the theoretical expected number of events.

The results of this analysis are shown in Fig. 3 for the nonuniversal parameters. We can see the complementarity between both reactor measurements and also notice that flux uncertainties related to the reactor antineutrino flux would not be the most relevant source of errors. To have a qualitative idea of the role of correlations, we have also computed the case in which the only source for systematics is a $2 \%$ error on each experiment, without any correlation. The result corresponds to the region surrounded by the dashed line in Fig. 3. As we can see, both regions are qualitatively similar, having the effect of a narrower constraint when the correlated errors are considered.

\section{CEVNS EXPERIMENTS WITH SPALLATION NEUTRON SOURCE}

Besides reactor antineutrinos, we can also make an analysis based on neutrinos coming from the SNS. That is the case of the COHERENT Collaboration, which used a CsI detector to make the first measurement of CEvNS. Here, a relatively high energy proton beam collides with a mercury target to produce high energy neutrons. As a result of the interaction, there is also a production of electron neutrinos, muon neutrinos, and muon antineutrinos, each with a distribution of the form,

$$
\begin{aligned}
& \frac{\mathrm{dN}_{\nu_{\mu}}}{\mathrm{d} E}=\eta \delta\left(E-\frac{m_{\pi}^{2}-m_{\mu}^{2}}{2 m_{\pi}}\right), \\
& \frac{\mathrm{dN}_{\bar{\nu}_{\mu}}}{\mathrm{d} E}=\eta \frac{64 E^{2}}{m_{\mu}^{3}}\left(\frac{3}{4}-\frac{E}{m_{\mu}}\right), \\
& \frac{\mathrm{dN}_{\nu_{\mathrm{e}}}}{\mathrm{d} E}=\eta \frac{192 E^{2}}{m_{\mu}^{3}}\left(\frac{1}{2}-\frac{E}{m_{\mu}}\right),
\end{aligned}
$$

where $\eta=r N_{\mathrm{POT}} / 4 \pi L^{2}$, a normalization factor with $r=0.08$ being the number of neutrinos per flavor; $N_{\text {POT }}=1.76 \times 10^{23}$, the number of protons on target, and $L$, the distance from the source to the detector. The total neutrino flux will be given by the sum of the three contributions. In contrast to neutrinos coming from nuclear reactors, these neutrinos have a maximum energy of $\sim 52 \mathrm{MeV}$, and, in consequence, the form factors are no longer constant, and they play an important role in the computation of the cross section. This means that the experimental data coming from this measurement are sensitive to nuclear information (see below) as discussed in Ref. [16]. In this section, we will simultaneously study the nuclear neutron distribution of iodine together with the possibility of having NSI interactions in the process. First, we will make the analysis using the currently available data from the CsI detector, and then we will give a future perspective for the NaI proposal [83].

Once the neutrino flux has been specified, we can compute the expected number of events, which for this experiment is given by

$$
N^{t h}=N_{D} \int_{T} A(T) d T \int_{E_{\min }}^{52.8 \mathrm{MeV}} d E \sum_{a} \frac{\mathrm{d} N_{a}}{\mathrm{~d} E} \frac{\mathrm{d} \sigma_{a}}{\mathrm{~d} T},
$$


with $N_{D}$, the number of targets within the detector, and $A(T)$, an acceptance function. The nuclear recoil energy $T$ has a maximum value which is well approximated by $T_{\max }\left(E_{\nu}\right) \simeq 2 E_{\nu}^{2} / M$, while the minimum value is specified by the detector properties. The sum runs over the three different neutrino fluxes, each multiplied by the corresponding cross section, which can be either the standard or the nonstandard one depending on the NSI parameters under study. Also, for all our SNS calculations, we have set the same time window as the one reported by the COHERENT Collaboration in [2]. As mentioned before, this neutrino counting experiment is sensitive to NSI parameters and to the neutron nuclear distribution. The former explicitly through the cross section in Eq. (3) and the latter through the form factors in the same equation. It has been shown that the effect of the form factor in the theoretical number of events is model independent [16], and thus, we will consider both proton and neutron distributions as given by a symmetrized Fermi one, that is [84],

$$
\begin{aligned}
F_{A}^{\mathrm{SF}}\left(q^{2}\right)= & \frac{3}{q c_{A}\left[\left(q c_{A}\right)^{2}+(\pi q a)^{2}\right]}\left[\frac{\pi q a}{\sinh (\pi q a)}\right] \\
& \times\left[\frac{\pi q a \sin \left(q c_{A}\right)}{\tanh (\pi q a)}-q c_{A} \cos \left(q c_{A}\right)\right],
\end{aligned}
$$

with $A=Z, N$ indicating the form factor for protons and neutrons, respectively; $q^{2}=2 M T$, and, in both cases, $a=$ $0.5233 \mathrm{fm}$ is a parameter related to the surface thickness. On the other hand, the parameter $c_{A}$ contains information about the corresponding rms radius $R_{A}$ through the relation,

$$
R_{A}^{2}=\frac{3}{5} c_{A}^{2}+\frac{7}{5}(\pi a)^{2} .
$$

We can use this dependence to get constraints for the neutron rms radius of heavy isotopes on the target material. This can be done by minimizing the squared function,

$$
\begin{aligned}
\chi^{2}= & \left(\frac{N^{\exp }-(1+\alpha) N^{t h}(X)-(1+\beta) N^{b g}}{\sigma}\right)^{2} \\
& +\left(\frac{\alpha}{\sigma_{\alpha}}\right)^{2}+\left(\frac{\beta}{\sigma_{\beta}}\right)^{2}
\end{aligned}
$$

Here, $N^{\exp }$ represents the measured number of events, $N^{\text {th }}(X)$ corresponds to the predicted one as a function of a set of parameters $X, N^{b g}$ is the number of background events coming, for instance, from neutrino induced neutrons [85] and prompt neutrons [2], and $\alpha, \beta$ are parameters which quantify systematic and background errors with their corresponding uncertainty.

Based on the previous discussion, we present our results. First, we will present our obtained constraints from the published CsI COHERENT data [2]. To this end, by following the approach in Ref. [16], we will consider
Cs and I nuclei as indistinguishable, with their corresponding proton distributions as is well known. Besides, the acceptance function in Eq. (19) will be given by

$$
A(x)=\frac{a}{1+\exp \left(-k\left(x-x_{0}\right)\right)} \Theta(x-5),
$$

where $\Theta$ is a Heaviside modified function and $a, k$, and $x_{0}$ are fixed parameters [86]. The argument $x$ (number of photoelectrons) in Eq. (23) satisfies $x \propto Q_{f}\left(T_{A}\right) T_{A}$, with $Q_{f}$ the quenching factor, defined as the nuclear recoil energy fraction, compared to an electron recoil. Initially, this quenching factor (QF) was considered by the COHERENT Collaboration as constant [2], but recent studies have proposed a behavior as a function of the nuclear recoil energy as shown in Ref. [87]. For our calculations, we will consider both pictures and see how the results are affected by the choice of QF.

Besides being sensitive to $R_{n}$, notice that the cross section in (3) also depends on NSI parameters. This means that any experimentally measured deviation from the SM number of events in Eq. (19) can be attributed to a lack of knowledge of the nuclear distribution of the Cs and I nuclei or to new physics. Motivated by this last statement, and as a first approach, we will consider one of the NSI parameters, $\epsilon_{e e}^{d V}$, as nonzero, and we will constrain it together with $R_{n}$. That is, we will minimize Eq. (22) with $X=\left\{\epsilon_{e e}^{d V}, R_{n}\right\}$.

The analysis in this case was carried out by energy bins as in [16], with experimental data and background events, and their corresponding uncertainties, taken from [2]. The results are shown at $1 \sigma$ (region between blue lines) in Fig. 4, where a comparison is done for different QF. The left panel of Fig. 4 shows the results with the QF used by the COHERENT Collaboration, which implies $\sigma_{\alpha}=0.28$, whereas in the right panel, we used the QF proposed in [87], with $\sigma_{\alpha}=0.135$. In both cases, we took $\sigma_{\beta}=0.25$. Notice that the effect of taking a different $\mathrm{QF}$ is observed as a displacement in the allowed region.

In addition, motivated by the discussion in Sec. I, we can combine the results of nuclear reactor and SNS experiments to get better constraints to both parameters. To this end, we follow a simple approach for which the total $\chi^{2}$ function will be given by the sum of the individual ones, as usual for independent observables. Due to the low energy regime, the reactor measurements will be only sensitive to the NSI interactions. The results are also shown in the same left and right panels of Fig. 4, respectively, where we present the expected region of the combined analysis of the COHERENT data with the expected results from the CONUS experiment. In the first panel, the allowed region is consistent with Ref. [16], which corresponds to the standard case without NSI.

The complete plan of the COHERENT Collaboration includes a NaI detector to measure CEvNS. We will show what would be the expected impact on the previous results 

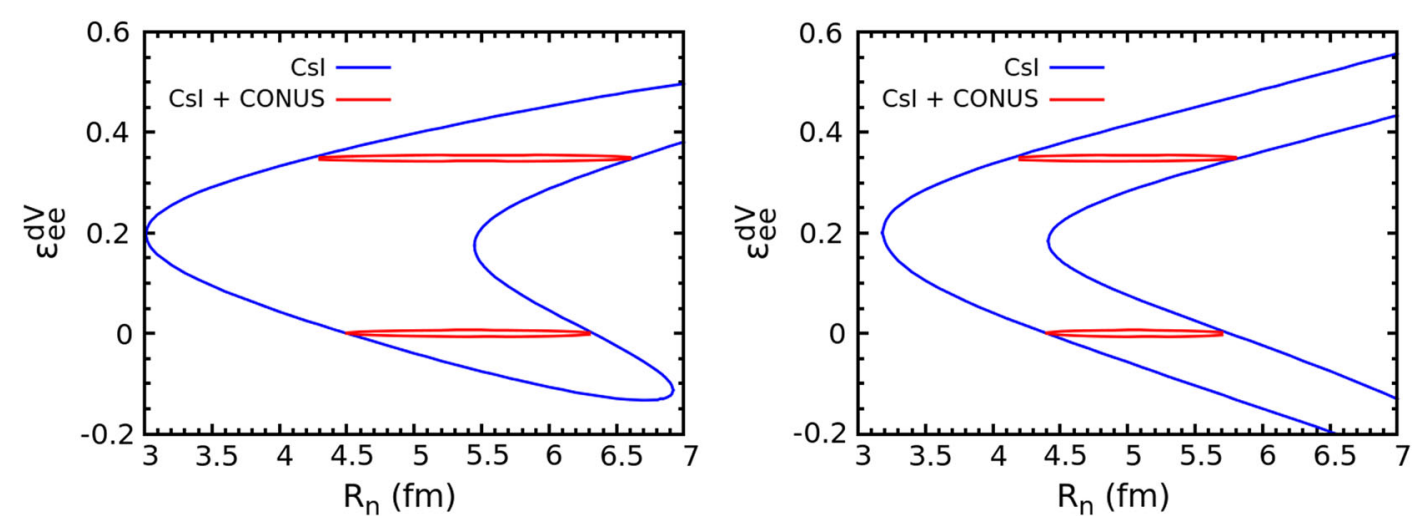

FIG. 4. Left panel: Allowed region from the CsI COHERENT data for the NSI parameter $\epsilon_{e e}^{d V}$ vs the mean neutron radius $R_{n}$ at $1 \sigma$. The original quenching factor reported by the collaboration was considered in this case. The restriction from a combined result with a future measure from the CONUS reactor experiment is illustrated with a red line. Right panel: same analysis as in the left panel, but considering now the recent reported new quenching factor (see text for details). In both panels, we have considered that reactors experiments will have an $8 \%$ systematic error.

by using this kind of detector. To this end, we follow a similar approach as for the CsI detector, but as we are talking about a future experiment, we need to make some assumptions. We will take the material detector mass, distance from the neutrino source, and threshold nuclear recoil energy as those given in Ref. [83], and regarding the acceptance function, we will consider the ideal case on which it is equal to one for all $T$; this is justified by the fact that the characterization of the $\mathrm{NaI}$ material has not been reported yet. Another important assumption is that we will take the experimental number of events in Eq. (19) to be the SM prediction considering a neutron rms radius of $5.5 \mathrm{fm}$, which corresponds to the minimum in the squared function when there are no NSI parameters involved and when a QF

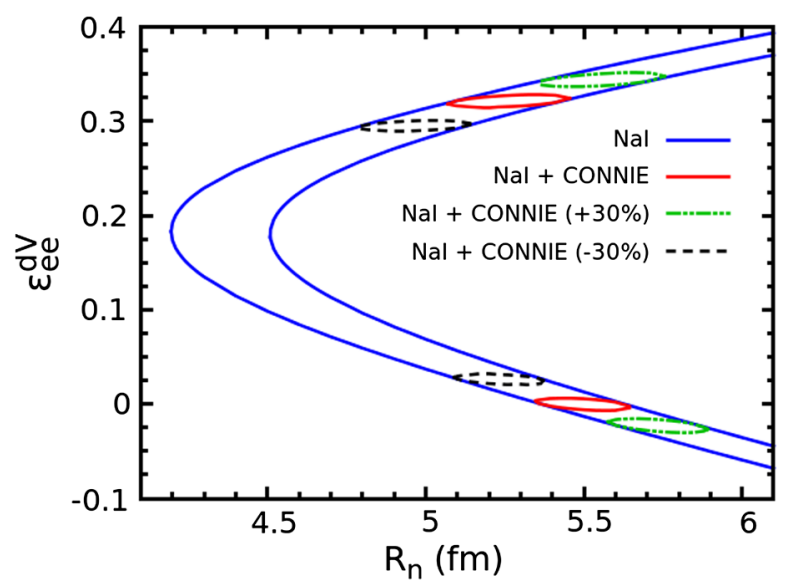

FIG. 5. Expected futuristic constraints from a NaI detector in combination with a CONNIE futuristic result at $1 \sigma$. In this case, we have considered different cases for the total measured events at CONNIE that illustrate how the combination of the two experiments can discriminate the values of both standard and nonstandard parameters. We have considered that reactors experiments will have an $8 \%$ systematic error. as that used by the COHERENT Collaboration is taken. Finally, we considered an optimistic case on which $\sigma_{\alpha}=$ 0.05 with $N^{b g}=10 \%$ of the SM prediction and $\sigma_{\beta}=0.10$.

The results are shown at a $1 \sigma$ level as the region between blue lines in Fig. 5. Following the previous analysis, we also show the expected region, combined with the CONNIE experiment analysis from Sec. III. In addition, we show the expected results assuming that the CONNIE experiment (the one with higher systematic uncertainties) reports a $\pm 30 \%$ deviation from the SM prediction. This assumption is only illustrative, so we can see that a considerable deviation from the SM prediction results in a displacement of the central value related to both the nuclear rms radius and the NSI parameter under study, but with the width of the errors unchanged. Notice that if CONNIE measures an excess above the SM prediction, the average radius should have a larger value, while the NSI signal will have negative values. In contrast, when the measured number of events for CONNIE is lower, the neutron radius takes lower values, and the NSI parameter prefers larger ones. This is consistent with the form factor dependence on the neutron radius and with the cross section linear and quadratic dependence on the nonuniversal NSI parameter $\epsilon_{e e}^{d V}$.

\section{CONCLUSIONS}

The increasing activity for a first detection of CEvNS with reactor antineutrinos and for an improved measurement of SNS CEvNS $[83,88]$ opens the door to future precise neutrino physics and to physics beyond the Standard Model constraints.

In order to have reliable constraints on physics beyond the Standard Model, we should have under control all possible standard contributions for a variation in the number of events, including the freedom in the nuclear physics parameters that appear in the neutron form factor. 
We have illustrated how the current measurement of CEvNS can be ascribed either to a correction in the neutron mean radius or to a variation in the NSI parameters. In the near future, reactor and SNS CEvNS measurements could be complementary and will help to improve the knowledge of nuclear and Standard Model parameters, constraining at the same time new physics, such as NSI parameters.

We have computed the expected sensitivity to NSI for different CEvNS proposals in reactor neutrinos and showed how, in combination with SNS experiments, they can contribute to have robust measurement of the mean neutron radius while improving NSI constraints at the same time.

\section{ACKNOWLEDGMENTS}

This work was supported by CONACYT-Mexico Grant No. A1-S-23238, SNI (Sistema Nacional de Investigadores), and PAPIIT Project No. IN115319. A. Parada was supported by Universidad Santiago de Cali (USC) under Grant No. 935-621118-3. B. C. Canas was supported by Universidad de Pamplona under Grant No. 400-156.012-027(GA313-BP-2019)
[1] D. Z. Freedman, Phys. Rev. D 9, 1389 (1974).

[2] D. Akimov et al. (COHERENT Collaboration), Science 357, 1123 (2017).

[3] D. K. Papoulias, arXiv:1907.11644.

[4] A. N. Khan and W. Rodejohann, Phys. Rev. D 100, 113003 (2019).

[5] P. Coloma, I. Esteban, M. C. Gonzalez-Garcia, and M. Maltoni, arXiv:1911.09109.

[6] D. K. Papoulias, T. S. Kosmas, and Y. Kuno, Front. Phys. 7, 191 (2019).

[7] D. Aristizabal Sierra, B. Dutta, S. Liao, and L. E. Strigari, J. High Energy Phys. 12 (2019) 124.

[8] O. G. Miranda, G. Sanchez Garcia, and O. Sanders, Adv. High Energy Phys. 2019, 3902819 (2019).

[9] W. Altmannshofer, M. Tammaro, and J. Zupan, J. High Energy Phys. 09 (2019) 083.

[10] M. Cadeddu, C. Giunti, K. A. Kouzakov, Y. F. Li, A. I. Studenikin, and Y. Y. Zhang, Phys. Rev. D 98, 113010 (2018).

[11] B. C. Cañas, E. A. Garcés, O. G. Miranda, and A. Parada, Phys. Lett. B 784, 159 (2018).

[12] J. Billard, J. Johnston, and B. J. Kavanagh, J. Cosmol. Astropart. Phys. 11 (2018) 016.

[13] P. B. Denton, Y. Farzan, and I. M. Shoemaker, J. High Energy Phys. 07 (2018) 037.

[14] Y. Farzan, M. Lindner, W. Rodejohann, and X.-J. Xu, J. High Energy Phys. 05 (2018) 066.

[15] D. K. Papoulias and T. S. Kosmas, Phys. Rev. D 97, 033003 (2018).

[16] M. Cadeddu, C. Giunti, Y. F. Li, and Y. Y. Zhang, Phys. Rev. Lett. 120, 072501 (2018).

[17] B. C. Cañas, E. A. Garcés, O. G. Miranda, and A. Parada, Phys. Lett. B 776, 451 (2018).

[18] S.-F. Ge and I. M. Shoemaker, J. High Energy Phys. 11 (2018) 066.

[19] E. Garces, B. Cañas, O. Miranda, and A. Parada, J. Phys. Conf. Ser. 934, 012004 (2017).

[20] I. M. Shoemaker, Phys. Rev. D 95, 115028 (2017).

[21] T. S. Kosmas, D. K. Papoulias, M. Tortola, and J. W. F. Valle, Phys. Rev. D 96, 063013 (2017).
[22] B. Dutta, Y. Gao, R. Mahapatra, N. Mirabolfathi, L. E. Strigari, and J. W. Walker, Phys. Rev. D 94, 093002 (2016).

[23] T. S. Kosmas, O. G. Miranda, D. K. Papoulias, M. Tortola, and J. W. F. Valle, Phys. Lett. B 750, 459 (2015).

[24] T. S. Kosmas, O. G. Miranda, D. K. Papoulias, M. Tortola, and J. W. F. Valle, Phys. Rev. D 92, 013011 (2015).

[25] J. Barranco, A. Bolanos, E. A. Garces, O. G. Miranda, and T. I. Rashba, Int. J. Mod. Phys. A 27, 1250147 (2012).

[26] J. Barranco, O. G. Miranda, and T. I. Rashba, Phys. Rev. D 76, 073008 (2007).

[27] J. Billard, L. E. Strigari, and E. Figueroa-Feliciano, Phys. Rev. D 91, 095023 (2015).

[28] C. Boehm, D. G. Cerdeno, P. A. N. Machado, A. OlivaresDel Campo, and E. Reid, J. Cosmol. Astropart. Phys. 01 (2019) 043.

[29] D. K. Papoulias, R. Sahu, T. S. Kosmas, V. K. B. Kota, and B. Nayak, Adv. High Energy Phys. 2018, 6031362 (2018).

[30] D. Aristizabal Sierra, N. Rojas, and M. H. G. Tytgat, J. High Energy Phys. 03 (2018) 197.

[31] H. T. Wong, H.-B. Li, J. Li, Q. Yue, and Z.-Y. Zhou, J. Phys. Conf. Ser. 39, 266 (2006).

[32] M. Lindner, W. Rodejohann, and X.-J. Xu, J. High Energy Phys. 03 (2017) 097.

[33] J. Rothe (NU-CLEUS Collaboration), Proc. Sci., NOW2018 (2018) 092.

[34] A. Aguilar-Arevalo et al. (CONNIE Collaboration), J. Instrum. 11, P07024 (2016).

[35] A. Aguilar-Arevalo et al. (CONNIE Collaboration), J. Phys. Conf. Ser. 761, 012057 (2016).

[36] M. Chávez-Estrada and A. A. Aguilar-Arevalo, J. Phys. Conf. Ser. 912, 012031 (2017).

[37] A. Aguilar-Arevalo et al. (CONNIE Collaboration), Phys. Rev. D 100, 092005 (2019).

[38] A. Aguilar-Arevalo et al. (CONNIE Collaboration), arXiv: 1910.04951.

[39] G. Agnolet et al. (MINER Collaboration), Nucl. Instrum. Methods A 853, 53 (2017).

[40] D. Yu. Akimov et al. (RED Collaboration), J. Instrum. 8, P10023 (2013).

[41] J. Billard et al., J. Phys. G 44, 105101 (2017). 
[42] X.-R. Huang and L.-W. Chen, Phys. Rev. D 100, 071301 (2019).

[43] D. Aristizabal Sierra, J. Liao, and D. Marfatia, J. High Energy Phys. 06 (2019) 141.

[44] Y. Farzan and M. Tortola, Front. Phys. 6, 10 (2018).

[45] O. G. Miranda and H. Nunokawa, New J. Phys. 17, 095002 (2015).

[46] T. Ohlsson, Rep. Prog. Phys. 76, 044201 (2013).

[47] P. S. Bhupal Dev et al., SciPost Phys. Proc. 2, 001 (2019).

[48] J. Barranco, O. G. Miranda, and T. I. Rashba, J. High Energy Phys. 12 (2005) 021.

[49] I. Esteban, M. C. Gonzalez-Garcia, M. Maltoni, I. MartinezSoler, and J. Salvado, J. High Energy Phys. 08 (2018) 180.

[50] D. Aristizabal Sierra, V. De Romeri, and N. Rojas, Phys. Rev. D 98, 075018 (2018).

[51] P. F. de Salas, D. V. Forero, C. A. Ternes, M. Tortola, and J. W. F. Valle, Phys. Lett. B 782, 633 (2018).

[52] Valencia-Globalfit, http://globalfit.astroparticles.es/ (2018).

[53] I. Esteban, M. C. Gonzalez-Garcia, M. Maltoni, I. MartinezSoler, and T. Schwetz, J. High Energy Phys. 01 (2017) 087.

[54] F. Capozzi, E. Lisi, A. Marrone, and A. Palazzo, Prog. Part. Nucl. Phys. 102, 48 (2018).

[55] O. G. Miranda, M. A. Tortola, and J. W. F. Valle, J. High Energy Phys. 10 (2006) 008.

[56] P. Coloma and T. Schwetz, Phys. Rev. D 94, 055005 (2016); 95, 079903(E) (2017).

[57] O. G. Miranda, M. Tortola, and J. W. F. Valle, Phys. Rev. Lett. 117, 061804 (2016).

[58] D. V. Forero and P. Huber, Phys. Rev. Lett. 117, 031801 (2016).

[59] A. Drukier and L. Stodolsky, Phys. Rev. D 30, 2295 (1984).

[60] K. Patton, J. Engel, G. C. McLaughlin, and N. Schunck, Phys. Rev. C 86, 024612 (2012).

[61] D. K. Papoulias and T. S. Kosmas, Adv. High Energy Phys. 2015, 763648 (2015).

[62] V. A. Bednyakov and D. V. Naumov, Phys. Rev. D 98, 053004 (2018).

[63] C. Patrignani et al. (Particle Data Group), Chin. Phys. C 40, 100001 (2016).

[64] K. Scholberg, Phys. Rev. D 73, 033005 (2006).

[65] J. B. Dent, B. Dutta, S. Liao, J. L. Newstead, L. E. Strigari, and J. W. Walker, Phys. Rev. D 97, 035009 (2018).
[66] P. Coloma, P. B. Denton, M. C. Gonzalez-Garcia, M. Maltoni, and T. Schwetz, J. High Energy Phys. 04 (2017) 116.

[67] D. K. Papoulias, T. S. Kosmas, R. Sahu, V. K. B. Kota, and M. Hota, Phys. Lett. B 800, 135133 (2020).

[68] P. Coloma, M. C. Gonzalez-Garcia, M. Maltoni, and T. Schwetz, Phys. Rev. D 96, 115007 (2017).

[69] O. G. Miranda, D. K. Papoulias, M. Tórtola, and J. W. F. Valle, J. High Energy Phys. 07 (2019) 103.

[70] A. Parada, arXiv:1907.04942.

[71] F. Reines and C. L. Cowan, Nature (London) 178, 446 (1956).

[72] C. L. Cowan, F. Reines, F. B. Harrison, H. W. Kruse, and A. D. McGuire, Science 124, 103 (1956).

[73] F. P. An et al. (Daya Bay Collaboration), Phys. Rev. Lett. 108, 171803 (2012).

[74] J. K. Ahn et al. (RENO Collaboration), Phys. Rev. Lett. 108, 191802 (2012).

[75] Y. Abe et al. (Double Chooz Collaboration), Phys. Rev. Lett. 108, 131801 (2012).

[76] M. Apollonio et al. (CHOOZ Collaboration), Phys. Lett. B 466, 415 (1999).

[77] M. Deniz et al. (TEXONO Collaboration), Phys. Rev. D 81, 072001 (2010).

[78] F. Reines, H. S. Gurr, and H. W. Sobel, Phys. Rev. Lett. 37, 315 (1976).

[79] T. Mueller, D. Lhuillier, M. Fallot, A. Letourneau, S. Cormon et al., Phys. Rev. C 83, 054615 (2011).

[80] P. Huber, Phys. Rev. C 84, 024617 (2011); 85, 029901(E) (2012).

[81] V. I. Kopeikin, L. A. Mikaelyan, and V. V. Sinev, Yad. Fiz. 60, 230 (1997) [Phys. At. Nucl. 60, 172 (1997)].

[82] P. Huber and T. Schwetz, Phys. Rev. D 70, 053011 (2004).

[83] D. Akimov et al. (COHERENT Collaboration), arXiv:1803 .09183 .

[84] J. Piekarewicz, A. R. Linero, P. Giuliani, and E. Chicken, Phys. Rev. C 94, 034316 (2016).

[85] E. Kolbe and K. Langanke, Phys. Rev. C 63, 025802 (2001).

[86] D. Akimov et al. (COHERENT Collaboration), arXiv:1804 .09459 .

[87] J. I. Collar, A. R. L. Kavner, and C. M. Lewis, Phys. Rev. D 100, 033003 (2019).

[88] D. Baxter et al., arXiv:1911.00762. 